\title{
Narrative review of the psychological impacts of lung cancer screening-consequences and how to address them?
}

\author{
Paloma Diab Garcia, Guido Van Hal \\ University of Antwerp, Antwerp, Belgium \\ Contributions: (I) Conception and design: Both authors; (II) Administrative support: Both authors; (III) Provision of study materials or patients: \\ Both authors; (IV) Collection and assembly of data: Both authors; (V) Data analysis and interpretation: Both authors; (VI) Manuscript writing: Both \\ authors; (VII) Final approval of manuscript: Both authors. \\ Correspondence to: Guido Van Hal. University of Antwerp, Universiteitsplein 1, 2610 Antwerp, Belgium. Email: guido.vanhal@uantwerpen.be.
}

\begin{abstract}
Lung cancer has a high mortality, mainly due to advanced stage diagnosis. However, when detected in an early stage, 5 -year survival rate can increase to $82 \%$. Primary prevention of lung cancer, by not smoking, is by far the best option to minimize the risk of getting the disease. Nevertheless, there are still a substantial number of people who are ex- or current smokers. For this group, and for people who have been exposed to carcinogenic substances, such as asbestos, secondary prevention—screening, could be life-saving. A lot of countries are already familiar for years with breast, cervical and colorectal cancer screening programmes. This is less the case for lung cancer screening (LCS). However, some countries already introduced LCS and the interest in it is growing. A big randomized, controlled trial in the USA and recently one in Europe, have shown that LCS might decrease the cause-specific mortality rate with one fifth to one fourth. Apart from the effectiveness of a screening programme in saving lives, however, there are also potential harms for participants. It is known from breast cancer screening, that psychological distress caused by taking part in a breast cancer screening programme, can last for a long period. For that reason, it is worthwhile to explore whether this would also be the case for LCS. In this review we look at possible psychological impacts of LCS. We found that false-positive and indeterminate results can lead to psychological distress, however, mostly in the short term. Without doubt, also stigmatisation will undeniably lay a huge psychological burden on participants. Psychological distress due to participating in LCS, should be minimised by taking measures which are as well screening-related, e.g., decreasing false-positive results as society-related, e.g., decreasing stigmatisation of lung cancer as a disease which is fully attributable to the smokers behaviour.
\end{abstract}

Keywords: Lung cancer screening (LCS); psychological consequences; participation; stigmatisation; false-positive results

Received: 10 February 2021; Accepted: 21 May 2021; Published: 30 October 2021.

doi: $10.21037 /$ shc-21-6

View this article at: http://dx.doi.org/10.21037/shc-21-6

\section{Introduction}

Lung cancer is the leading cause of cancer death, with $18 \%$ of worldwide cancer deaths (1) and with a 5-year relative survival rate that ranges from $63 \%$ in localised lung cancer to $7 \%$ for metastasised lung cancer (2).

This high mortality rate is mainly due to frequent advanced stage diagnosis (49-53\% at stage 4) (3). Studies have shown that the implementation of low dose computed tomography (LDCT) lung cancer screening (LCS) would allow for an earlier stage diagnosis which would increase the 5 -year survival rate from $6 \%$ (stage 4 diagnosis) to $82 \%$ (stage 1A diagnosis) (4). Two large studies took place to show the benefits of LCS in the high-risk population with one taking place in the United Stated (US National Lung Screening Trial-NLST) and the other in the Netherlands 
Table 1 Results showing the number of articles found when testing for different search terms during term search 1

\begin{tabular}{lcc}
\hline Search terms options & Number of articles (PubMed) & Number of articles (Web of Science) \\
\hline Psychology + lung cancer & 2,519 results & 64 results \\
lung cancer screening + consequences & 34 results & 38 results \\
Psychology + lung cancer + screening & 286 results & 14 results \\
Psychology + lung cancer + consequences & 77 results & 4 results \\
Psychology + lung cancer screening + consequences & 13 results & 1 result \\
\hline
\end{tabular}

Table 2 Results showing the number of articles found when testing for different search terms during term search 2

\begin{tabular}{lcc}
\hline Search terms options & Number of articles (PubMed) & Number of articles (Web of Science) \\
\hline Lung cancer screening+ consequences+ psychol ${ }^{*}$ & 1,594 & 18 \\
Lung cancer screening+ consequences+ psychological & 37 & 15 \\
Lung cancer screening+ consequences+ psychology & 67 & 1 \\
\hline
\end{tabular}

and Belgium (5). The NLST showed that LDCT fomented the reduction of the relative risk of lung cancer mortality by $20 \%$ which was later sustained by the NELSON who also identified a significant decrease but in this case by $26 \%(5-7)$.

Like for most cancers or chronic diseases many studies have taken place to determine its impact on quality of life. Nonetheless, since LCS programmes have not been widely implemented the knowledge on the psychological impact and consequences of such a screening is limited. Most studies tend to focus on the possible psychological impact of abnormal results and not so much on how the individual reacts and experiences LCS (8). This is a key area to investigate further as it can be relevant in the decision making of an individual to be willing to undergo LCS and influence recovery (9). Thus, it should not be overlooked as the impact of psychological harm could affect a large scale of the targeted individuals (10). This topic is even more important in a cancer like lung cancer which is significantly surrounded by stigmatisation (10). Therefore, if psychological harm occurs and it is added to the burden of the side-effects from the potential treatments this could have a negative impact on quality of life and could influence the decision of individuals to be screened (11).

Therefore, the aim of this review is to define the consequences of the psychological impact of lung cancer and the best method for clinicians to address them and even limit them during LCS programmes implementation.

We present the following article in accordance with the Narrative Review Reporting Checklist (available at: http:// dx.doi.org/10.21037/shc-21-6).

\section{Material and methods}

This review focused on studies which related to the consequences of the psychological impact that LCS can have on participants and how to address them. A first search was done to determine which word combinations would provide us with the most results (Table 1). Based on that first search the following search combinations (Table 2) were used to select the articles used in this review: "Lung cancer screening+ consequences+ psychological" (search term 1) and "Lung cancer screening+ consequences+ psychology" (search term 2).

It should be noted that we also decided to look on adding the search term "Lung cancer screening+ consequences+ psychol*". Nonetheless, due to the number of articles we got and from those the relevant ones being the ones we could find with search terms 1 and 2 we decided to discard it and not include. This search term yielded a very wide range of articles and many were being included that were not specific to our research question.

Due to lung screening being a recent phenomenon we did not have specific and clear-cut eligibility criteria. The only three main factors that influenced our article selection when screening based on title and abstract were that they be in a language we could read (French, Dutch, Spanish or English), that they focused on the psychological impact of the patient and not only the caregivers, and that they 

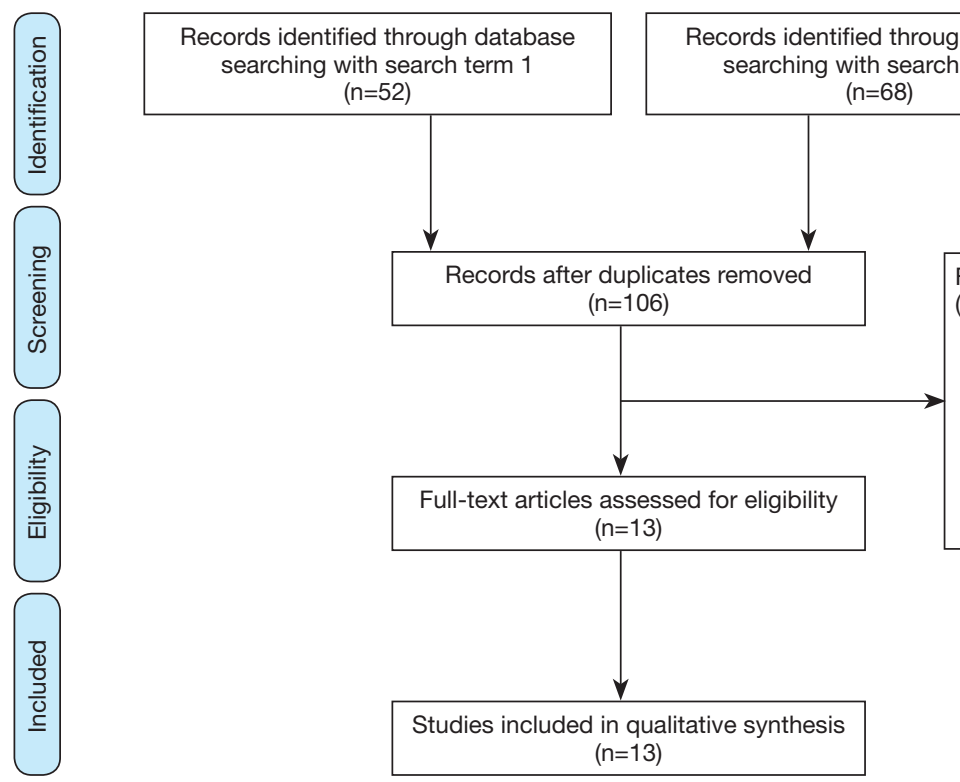

Full-text articles excluded, with reasons $(\mathrm{n}=93)$

Based on the title did not respond to our research question $(n=65)$

Based on abstract/text: Not focused on lung cancer screening/psychological impact, focus on caregivers' psychological impact instead of patients... $(n=26)$

Language that we could not understand (Polish and Danish) $(n=2)$

Figure 1 PRISMA flow diagram of selected articles in Web of Science and PubMed on "Lung cancer screening+ consequences+ psychological" (search term 1) AND "Lung cancer screening+ consequences+ psychology" (search term 2).

appropriately answered our research question. In Figure 1, the PRISMA flow diagram of selected articles in Web of Science and PubMed is presented (see Table S1 for the list of articles).

\section{Results}

From the selected articles, several relevant and frequent topics emerged as important. These topics will be used as headers for this results section.

\section{Psychological impact of screening programme structure}

\section{Informed decision making}

Part of the NELSON trial focused on evaluating the impact of LCS on the health-related quality of life (HRQoL). More specifically if informed decision making on LCS could be associated with a better HRQoL (12).

Based on this study van den Bergh et al. concluded that no association could be observed between informed decision making and a betterment in HRQoL independently of the screening results. Therefore, suggesting that the impact of informed decision making on the psyche of the individual is not significant (12).

Nonetheless, these results should be looked at with some caution as this data is based on the decision to participate in a trial and not in a real screening programme. Therefore, not being a basis to promote uninformed decision making of potential individuals to be screened and more research should be done in real clinical settings to confirm these findings.

\section{Screening process}

During the NLST trials, there was some concern on the potential psychological burden of LDCT screening on individuals ${ }^{7}$. Many studies have later focused on the psychological impact of abnormal results instead of on the individual response. Nonetheless, in the last few years research has started to focus on the individual and has shown that different social, cognitive, and attitudinal response can be observed in those that undergo screening contrary to those that do not (8).

Based on the latter mentioned findings Kummer et al., decided to focus on the psychological impact at the individual level of the different parts of the screening process and came to the following conclusions. Prior to the lung health check and when told about eligibility a decrease in worry and anxiety could be seen in individuals as it provided them with a sense of control of their health as it allowed them to address any concerns about undiagnosed symptoms (8). The start of the negative psychological impact was established during the spirometry test as 
it set the tone for the rest of the screening process (8). Nonetheless, although the latter sets the tone the peak moment of extreme anxiety seen in individuals is during the waiting time for the LDCT results (8).

This conclusion has been corroborated in other studies like the NELSON study which showed that $46 \%$ of participants reported discomfort during that time and $50 \%$ dreaded the results, with distress levels rising even more two months later for those with indeterminate results (13). This fear of not knowing what to expect and the seriousness of a positive result, results in a significant psychological burden more specifically extreme anxiety burden. Thus, affecting the HRQoL.

Psychological preparedness is key to decrease the psychological burden during the screening test specifically when receiving results. As previously mentioned, those with indeterminate results experience continuous anxiety, worry and fear of cancer. Although there is an important psychological burden, it seems like preparing one's self for the worse lessened the adverse psychological outcomes contrary to those that did not (8).

While one could say based on the information summarised previously that in terms of psychological burden the harms outweigh the benefits of screening, it is not completely true. It can be agreed that the waiting of results and more specifically indeterminate results can lead to high levels of stress, but it is just temporary. The possibility of a follow up test provides reassurance to the patient enabling a decrease in anxiety and when asked if participants would undergo screening again the responses were favourable especially in smokers as it provided them with some peace of mind (14).

\section{Types of LDCT results}

As previously mentioned at the beginning of this section of the research many studies on the psychological burden of LCS have focused on the type of screening results. Byrne et al., and Dalphin JC, concluded that indeterminate results translate in significant anxiety and worry from the patient. While those with a negative result had a significant decrease in negative effects associated with the feeling of relief $(15,16)$. This finding is important when considering that spiral CT screening has a range of $23-51 \%$ of suspicious/ indeterminate results (15). McGovern et al., through a qualitative study determined that abnormal screens and diagnostics resulted in anxiety, fear of cancer and death, and emotional distress as well. Nonetheless, the research also showed that these negative psychological consequences tend to decrease over time (17). Distress levels tend to stay high until the results of the follow up scans are known, or individuals had a consultation with a specialist (15). Therefore, adverse psychological burden could be characterised as short term and emotional but positive for the majority in the long term $(13,14,16)$. Thus, not significantly impacting HRQoL in the long term.

Moreover, the Pittsburgh Lung Screening Study (PLuSS) defined the period of heightened negative psychological effect during screening to be 1-2 weeks after abnormal or positive results (13). They also determined the type of emotional reaction based on gender, level of education and social and smoking status. Current smokers had higher levels of anxiety and fear of cancer than non/ex-smokers (13). Women reported higher levels of fear of cancer while married participants and those with a high education level tended to report lower levels of anxiety (13). Nonetheless general distress tended to decrease after one year to baseline levels like those with a negative result (13).

Another aspect of the research that should be highlighted is that although it is important to inform an individual during the decision-making process it should be done in a non-fatalistic way. Too much emphasis on the high rates of false positives and potential negative psychological effects of screening might be doing more harm than good (15). Thus, increasing the fatalistic perception of the individual and increasing the rate of negative psychological effects, which might alter individual's decision to be screened (15). The impact of false positives on compliance to screening has been source of debate as some studies have shown that the impact is non-significant (16). Nonetheless, considering the level of debate around this subject, the main recommendation would be to continue to inform while being conscious on how the information is being transmitted and on the reaction of the individual receiving this information.

\section{Psychological impact based on perception of risk by individual and society}

\section{Individual self-assessment of risk}

While analysing the NLST data collected on psychological burden of LDCT screening Kummer et al., identified that the intensity and type of individual psychological response is determined by personal cognitive risk factors (8). More specifically a higher affective risk perception and presence of self-blame (8). These conclusions are in accordance with the study done by Byrne et al. (15). Contrary to some 
research that suggests that those with a higher risk to test positive for lung cancer underestimate their risk, his study showed that it was overestimated (15). This result was not exclusive to smokers, all participants no-matter their smoking status tended to overestimate their risk of lung cancer before getting the results, leading to a generalised increase in anxiety (15). Although, it is true that those levels will be different depending on smoking status and the highest will tend to be seen in those having known risks that will elevate the chances of a positive diagnosis.

\section{Asbestos risk}

As it was stated previously level of anxiety and psychological burden will depend on the presence of known risk factors. Although, smoking is the main one known and most common another one is also asbestos exposure. Maurel et al., studied a group of asbestos exposed subjects who were screened and concluded that significant higher levels of negative psychological consequences could be observed contrary to the control group (18). Barak et al., assessed 13 workers who were exposed to asbestos and underwent screening. He observed that $50 \%$ were diagnosed with post-traumatic stress disorder due to a phenomenon of anticipation of death during the psychological assessment (19). In line with these findings Maurel et al., described as severe the psychological burden associated to the screening of asbestos exposed individuals. Moreover, a strong correlation was established between the level of asbestos distress and the individual self-perception of the level of asbestos exposure and self-reported asbestos related disease (18). Paris et al. utilised a validated psychological consequences questionnaire on a cohort of 832 retired asbestos exposed workers to determine impact of CT screening. The conclusions made although in line with previous studies are more specific. A significant increase of the psychological score was observed till 6 months after baseline in participants with abnormal and normal scans (20). The type of finding also determined the level of anxiety. The detection of isolated pulmonary nodules which is often associated with potential more serious health consequences translate in a significant increase in feelings of distress (20). This phenomenon affects a considerable percentage of asbestos exposed individuals specifically at the start of the screening as they tend to be more aware about their health and any possible physical changes (18).

Therefore, more fatalistic thoughts, severe anxiety and fear of death and possible post-traumatic stress disorder can occur even 6 months after results were given. This information is key when screening this specific group as the psychological negative effects although similar to smokers are much more severe and are long term instead of short term.

\section{Stigma}

As we have seen so far psychological burden can affect a large portion of patients. In lung cancer this is magnified contrary to other cancers due to the stigma surrounding this type of disease which is mainly found in smokers. There is the belief that it is the fault of the patient as they are the ones that chose to smoke knowing the risk of cancer associated to this behaviour. In his research, DeFrank et al., observed that although labelling with a condition usually results in positive health effects-positive lifestyle changes, negative effects are also possible. This is particularly important in lung cancer as labelling is an important source of potential harm of screening and brings about negative changes (anxiety, altered self-concept, stigma...) (10).

Kummer et al., also noticed this association between stigma and smoking when studying the psychological data collected during the NLST study. In smokers stigma led to an increase in worry about potential LDCT results, higher affective risk perception and lack of reassurance that LDCT results will be negative (8). This stigma also fomented in smokers' fatalistic attitudes and more negative views on their health (8). Moreover, in some generations in which the stigma surrounding smoking and lung cancer is higher the perception that lung cancer is a death sentence is much higher (8). Stigma does not only alter their emotional status but also makes believe them that no one will support them and thus will not ask for any social support or even disclose their results (8).

Therefore, making the psychological burden that much important and having a major effect on HRQoL as they have no support which is crucial in any type of chronic disease like cancer. Thus, dealing with stigma must be one of the major focuses by clinicians and policy makers during a screening programme.

\section{Social support and behaviour change}

When dealing with a life alternating chronic disease social support can be key for HRQoL and to foment positive behavioural change. Social support is not only important after diagnosis but also during the screening process to deal with all the uncertainty and anxiety surrounding the process (8). Having a good social support can be used as a type of buffering system (8). This buffering can be defined 
as emotional support, aiding in comprehension of LDCT results and supporting positive behaviour change (8). Moreover, social support can also be found in others participating in trials who have had negative results, thus providing some reassurance (21).

Participating in screening apart from providing a peace of mind and some control led to positive health changes especially in the presence of a good social support. Some of the changes commonly seen include changes in the smoking habits (cutting down and in some cases smoking cessation) and engaging more frequently in general cancer prevention behaviour (exercise, change in diet, avoiding air pollution and going to the GP regularly for lung capacity checks) (8). Screening programmes and providing information on which symptoms to look for also leads to greater attention of individuals and consciousness to their health especially in those at higher risk (smokers, asbestos exposure...) $(8,20)$. There is a greater regularity in GP visits and a faster seeking of help if symptoms present (8).

Therefore, screening gives the tools for those with negative results and at high risk to regain some control on their condition and health fomenting the reduction of anxiety and worry. Thus, the negative psychological burden to give way to more positive ones.

\section{Psychological harm}

Through this review we have seen that psychological harm from screening affects all in different ways and can range from mild to severe (anxiety, distress, dejection, and decrements in HRQoL...) during different aspects of the screening process $(10,21,22)$. Although, many studies have suggested a high denial risk in high-risk individuals like smokers Maurel et al. (18) and other similar studies have disproved this. Showing that there is more to it, that denial is not that common and even if it is present there is still some psychological burden (19). Additionally, that in some high-risk groups like asbestos exposure the denial of risk is non-existent explaining the high levels of anxiety surrounding LCS (18).

The NELSON study further demonstrated that the psychological burden starts as well before the screening took place and differs based on level of risk (10). Thus, supporting the idea that denial is not high in high-risk groups. $14.6 \%$ of participants reported a personal high affective risk a day before screening with significant worse levels of distress and general HRQoL than those reporting low affective risk (14). Moreover, Wu et al., further supported the research concluding that psychological harm is short term contrary to other cancer screening programmes like for breast cancer (14).

Nonetheless, it should be noted that the psychological harm determined in most studies is based on screening trials which limits the validity of the true psychological burden. More high-quality research is needed to determine the frequency, duration and overall magnitude of LCS related psychological burden in non-clinical settings (14).

\section{How to address these consequences?}

Throughout the three previous parts we determined which were the psychological harms and which factors influenced their frequency and severity. In this section we will now focus more on the suggestions given by the different researchers to deal with these consequences.

One of the most common suggestions is to provide information about psychological harm (10). Firstly, information should not only focus on possible negative psychological consequences but also provide a clear and detailed explanation of the meanings of the different types of results and about an individual's objective risk before screening (15). Moreover, for this transmission of knowledge to be effective it should be made clear to clinicians that psychological harm might be significant due to high burden, frequency, or both (10). Therefore, less frequent harms but with a high burden and vice versa should not be considered as trivial (8). The tone and amount of information should also be considered as too much or too little might affect adherence to screening, finding the right balance is crucial $(12,21)$. An alternative way of transmitting the information and which has shown to significantly lower anxiety and promote psychological preparedness for LCS (reduce distress) is a five-minute video intervention which provides information on screening criteria, procedures, benefits and harms, and follow-up plan (22). This video is accompanied by a nine-page handbook and the possibility to contact a LCS staff member to ask any follow-up questions that might surge (22). Also, providing information about the small risk of having lung cancer in the invitation letter for screening might enable a decrease in distress (23).

Secondly, due to the stigmatisation surrounding lung cancer patients and to promote positive behavioural change a broader approach should be used. Specifically, instead of the cancer prevention programme focusing on just smoking cessation a broader cancer prevention approach should be used as many high-risk behaviours are shared between 
Table 3 Several ways to deal with the psychological burden of participating in lung cancer screening

Psychological preparedness (preparing one's self for the worse)

Possibility of a follow up test or consultation with a specialist

Balanced information, not only emphasizing the potential negative psychological effects of screening, possibly with a video

Dealing with stigma

Social support

A broad cancer prevention approach

Psychological intervention through a medical survey

cancers (8).

Thirdly, for high-risk individuals particularly (could also be used on low-risk ones) a psychological intervention through a medical survey would be of great benefit (18). By addressing the mental well-being of those undergoing screening it would provide clinicians the information they need to give the right tools and increase the sense of control that patients lack during this process (18). Moreover, it provides another level of support which is more professional and decreases potential harm. To achieve the most effective impact the questionnaires used to test for HRQoL should include screening specific questions which would provide quantitative data to determine if factors like spiritual faith, satisfaction with health care and social support mediate the impact of screening in HRQoL (16). Also, the LCS specific questions should have high content validity and adequate psychometric properties (20).

Lastly, another important consequence is the significant impact high false positive rates can have on the psychological burden. Effort should focus on minimising this rate by carefully considering eligibility criteria, optimising the balance of sensitivity versus specificity of LDCT result reporting and following diagnostic algorithms (13). Clinicians can also tailor communication strategies that can decrease distress and emphasise adequate information exchange, consideration of values and preferences, and shared decision making (13). Also, identifying a subset of nodules with an increased cancer risk or by combining imaging and proteomics or genomic biomarkers might allow for a decrease in false positives (23).

In summary, development, and further refinement of a conceptual framework on the psychological burden of LCS can potentially yield more high-quality evidence in future research (14). Thus, finding the most optimal way to address and minimise psychological burden of screening. In Table 3, a summary is presented of several ways to deal with the psychological burden of participating in LCS.

\section{Discussion}

Lung cancer is the most commonly occurring cancer in men and the third most commonly occurring cancer in women. Globally, there were 2.1 million new cases and 1.8 million deaths in 2018 (24). It could be expected that with this high mortality the psychological burden on patients undergoing screening will be significant, which we aimed to determine through this study.

Throughout the literature review performed we were able to identify the extent of the psychological burden, as well as which groups' mental well-being should be supervised more and some techniques and suggestions to best deal with and prevent these burdens. Although the burden is significant, we have to emphasise that they are mostly only short-term contrary to other cancers like breast cancer (14). The psychological burden tends to start right after the spirometry test and is at its highest during the results waiting time (6). This burden can be characterised as being emotional more than physical and includes anxiety, distress, fear of cancer and death, and worry (8). Although some research has suggested that individuals eligible for screening especially those in high-risk groups tend to underestimate their risk, this review concludes otherwise. Various studies have demonstrated an overestimation in all groups screened of their risk and that overestimation is even higher in high-risk groups like smokers or in people exposed to asbestos (15). The latter even show much higher levels of psychological burden with those being exposed to asbestos presenting post-traumatic stress disorder after screening (15). More attention should be paid to those 
groups mental health and wellbeing, making sure they have an objective idea of their true risk to decrease their fatalistic thoughts and anxiety levels.

Although screening those groups brings about an important amount of negative psychological effects it also brings about positive ones like more health awareness, faster GP seeking and more regular visits, change in smoking habits, increase in cancer prevention behaviour and a sense of control over their health and some relief at the end of the process $(8,20)$.

To allow the positive to outweigh the negative various techniques can be used. Using informing videos and giving an opportunity to patients to talk with a professional to address their doubts before screening. Clinicians should provide the necessary information both about what the different results mean and individuals risk in a balanced manner as not to increase anxiety level further and decrease fatalistic thoughts. They should be aware of the different types of psychological burdens possible and during the screening process provide the tools necessary to minimise them especially to those in high-risk groups. Furthermore, before the screening process it would be optimal to make sure the individual has a good social support or provide alternatives like other people in the programme and minimise social stigmatisation. The latter can be done through the collaboration with policy makers and media communication. Social support and low stigmatisation are key to promote and motivate positive behavioural change and a decrease in negative psychological effects. Another key factor is minimising the rate of false positives by identifying a clear screening group and using different techniques and informing appropriately about the meaning and implications of a false positive test.

This review is based on studies which used a variety of research methods, study designs and sample sizes. Besides of three systematic reviews, there was a narrative article, an RCT, an intervention study, quantitative studies with questionnaires and a qualitative study with seven focus groups. Regarding the questionnaire studies, most used selfadministered questionnaires but one study conducted semistructured interviews. Some studies mentioned they used validated questionnaires, other did not. A few studies carried out the questionnaire in the same group of respondents on several moments in time, others did a mere cross-sectional study. Also regarding the sample size, marked differences could be seen, from semi-structured interviews in 28 current and former smokers, to a few thousands respondents in a study with self-administered questionnaires. Several studies included validated questionnaires, as well regarding the psychological consequences as regarding the setting of LCS. Others did not mention this. Although all studies included in our review can be considered as being of good quality, some general remarks should be made in view of future research. At this moment, the number of studies on the topic of LCS and its psychological consequences on the participants, is scarce. Moreover, sometimes, more appropriate measurement tools could be used, for instance for taking into account the specific situation of LCS or by using validated scales to measure psychological consequences. Most of the time, studies were conducted within a trial setting and only a few studies did follow-up the respondents prospectively. Studies should also be more based on representative samples instead of on convenience samples.

\section{Conclusions}

When we want LCS programmes to be a success and increase participation an important aspect should be to promote a positive mental well-being or at least minimise the negative psychological burden. Throughout the review we have established some of these burdens and how to deal with them or minimise them. Nonetheless, there is still a lot of work to do. More research should take place in nonclinical settings to determine the true psychological impact and if the measures suggested are truly effective. Moreover, work should not only focus on educating clinicians on psychological burden or providing patients with the right tools it should also focus on educating the population. The education of the population on lung cancer is extremely important to decrease stigmatisation which undeniably has a huge psychological burden on participants. Also, more research should focus on individual psychological response to the screening process and not only on abnormal results. We still have a long way to go but hopefully through the start of LCS programmes in various countries we will be able to get a better picture and make this whole process more efficient in terms of mental well-being.

\section{Acknowledgments}

Funding: None.

\section{Footnote}

Provenance and Peer Review: This article was commissioned by the Guest Editors (Wieland Voigt and Helmut Prosch) 
for the series "Lung Cancer Screening" published in Shanghai Chest. The article has undergone external peer review.

Reporting Checklist: The authors have completed the Narrative Review Reporting Checklist. Available at: http:// dx.doi.org/10.21037/shc-21-6

Peer Review File: Available at http://dx.doi.org/10.21037/ shc-21-6

Conflicts of Interest: Both authors have completed the ICMJE uniform disclosure form (available at http:// dx.doi.org/10.21037/shc-21-6). The series "Lung Cancer Screening" was commissioned by the editorial office without any funding or sponsorship. The authors have no other conflicts of interest to declare.

Ethical Statement: The authors are accountable for all aspects of the work in ensuring that questions related to the accuracy or integrity of any part of the work are appropriately investigated and resolved.

Open Access Statement: This is an Open Access article distributed in accordance with the Creative Commons Attribution-NonCommercial-NoDerivs 4.0 International License (CC BY-NC-ND 4.0), which permits the noncommercial replication and distribution of the article with the strict proviso that no changes or edits are made and the original work is properly cited (including links to both the formal publication through the relevant DOI and the license). See: https://creativecommons.org/licenses/by-nc-nd/4.0/.

\section{References}

1. Sung H, Ferlay J, Siegel RL, et al. Global Cancer Statistics 2020: GLOBOCAN Estimates of Incidence and Mortality Worldwide for 36 Cancers in 185 Countries. CA Cancer J Clin 2021;71:209-49.

2. American Cancer Society. Lung Cancer Survival Rates. Accessed on 5th May, 2021. Available online: https:// www.cancer.org/cancer/lung-cancer/detection-diagnosisstaging/survival-rates.html

3. Cancer Research UK. Lung cancer incidence statistics. Accessed May 13, 2019. Available online: https://www. cancerresearchuk.org/health-professional/cancer-statistics/ statistics-by-cancer-type/lung-cancer/survival\#headingThree
4. Goldstraw P, Chansky K, Crowley J, et al. The IASLC lung cancer staging project: proposals for revision of the TNM stage groupings in the forthcoming (eighth) edition of the TNM classification for lung cancer. J Thorac Oncol 2016;11:39-51.

5. de Koning HJ, van der Aalst CM, de Jong PA, et al. Reduced Lung-Cancer Mortality with Volume CT Screening in a Randomized Trial. N Engl J Med 2020;382:503-13.

6. Aberle DR, Berg CD, Black WC, et al. The National Lung Screening Trial: overview and study design. Radiology 2011;258:243-53.

7. Aberle DR, Adams AM, Berg CD, et al. Reduced lung cancer mortality with low-dose computed tomographic screening. N Engl J Med 2011;365:395-409.

8. Kummer S, Waller J, Ruparel M, et al. Mapping the spectrum of psychological and behavioural responses to low-dose CT lung cancer screening offered within a Lung Health Check. Health Expect 2020;23:433-41.

9. Chapple A, Ziebland S, McPherson A. Stigma, shame, and blame experienced by patients with lung cancer: qualitative study. BMJ 2004;328:1470.

10. DeFrank JT, Barclay C, Sheridan S, et al. The psychological harms of screening: the evidence we have versus the evidence we need. J Gen Intern Med 2015;30:242-8.

11. Danson SJ, Rowland C, Rowe R, et al. The relationship between smoking and quality of life in advanced lung cancer patients: a prospective longitudinal study. Support Care Cancer 2016;24:1507-16.

12. van den Bergh KA, Essink-Bot ML, van Klaveren RJ, et al. Informed decision making does not affect health-related quality of life in lung cancer screening (NELSON trial). Eur J Cancer 2010;46:3300-6.

13. Slatore CG, Sullivan DR, Pappas M, et al. Patientcentered outcomes among lung cancer screening recipients with computed tomography: a systematic review. J Thorac Oncol 2014;9:927-34.

14. Wu GX, Raz DJ, Brown L, et al. Psychological Burden Associated With Lung Cancer Screening: A Systematic Review. Clin Lung Cancer 2016;17:315-24.

15. Byrne MM, Weissfeld J, Roberts MS. Anxiety, fear of cancer, and perceived risk of cancer following lung cancer screening. Med Decis Making 2008;28:917-25.

16. Dalphin JC. Quels sont les objectifs du suivi postprofessionnel, les bénéfices attendus et les risques possibles? Objectifs médicaux en termes de morbidité, mortalité et qualité de vie [Follow-up of subjects 
occupationally exposed to asbestos, what are the objectives, the benefits, and the possible risks?]. Rev Mal Respir 2011;28:1230-40.

17. McGovern PM, Gross CR, Krueger RA, et al. FalsePositive Cancer Screens and Health-related Quality of Life. Cancer Nursing 2004;27:347-52.

18. Maurel M, Stoufflet A, Thorel L, et al. Factors associated with cancer distress in the Asbestos Post-Exposure Survey (APEXS). Am J Ind Med 2009;52:288-96.

19. Barak Y, Achiron A, Rotstein Z, et al. Stress associated with asbestosis: The trauma of waiting for death. Psychooncology 1998;7:126-8.

20. Paris C, Maurel M, Luc A, et al. CT scan screening is associated with increased distress among subjects of the APExS. BMC Public Health 2010;10:647.

21. Rasmussen JF, Siersma V, Pedersen JH, et al.

doi: $10.21037 /$ shc-21-6

Cite this article as: Diab Garcia P, Van Hal G. Narrative review of the psychological impacts of lung cancer screeningconsequences and how to address them? Shanghai Chest 2021;5:38.
Psychosocial consequences in the Danish randomised controlled lung cancer screening trial (DLCST). Lung Cancer 2015;87:65-72.

22. Raz DJ, Nelson RA, Kim JY, et al. Pilot study of a video intervention to reduce anxiety and promote preparedness for lung cancer screening. Cancer Treat Res Commun 2018;16:1-8.

23. van den Bergh KA, Essink-Bot ML, Borsboom GJ, et al. Short-term health-related quality of life consequences in a lung cancer CT screening trial (NELSON). Br J Cancer 2010;102:27-34.

24. World Cancer Research Fund International. Lung cancer statistics. Accessed on 5th of May, 2021. Available online: https://www.wcrf.org/dietandcancer/lung-cancerstatistics/ 
Supplementary

Table S1 List of articles used after the selection process was completed

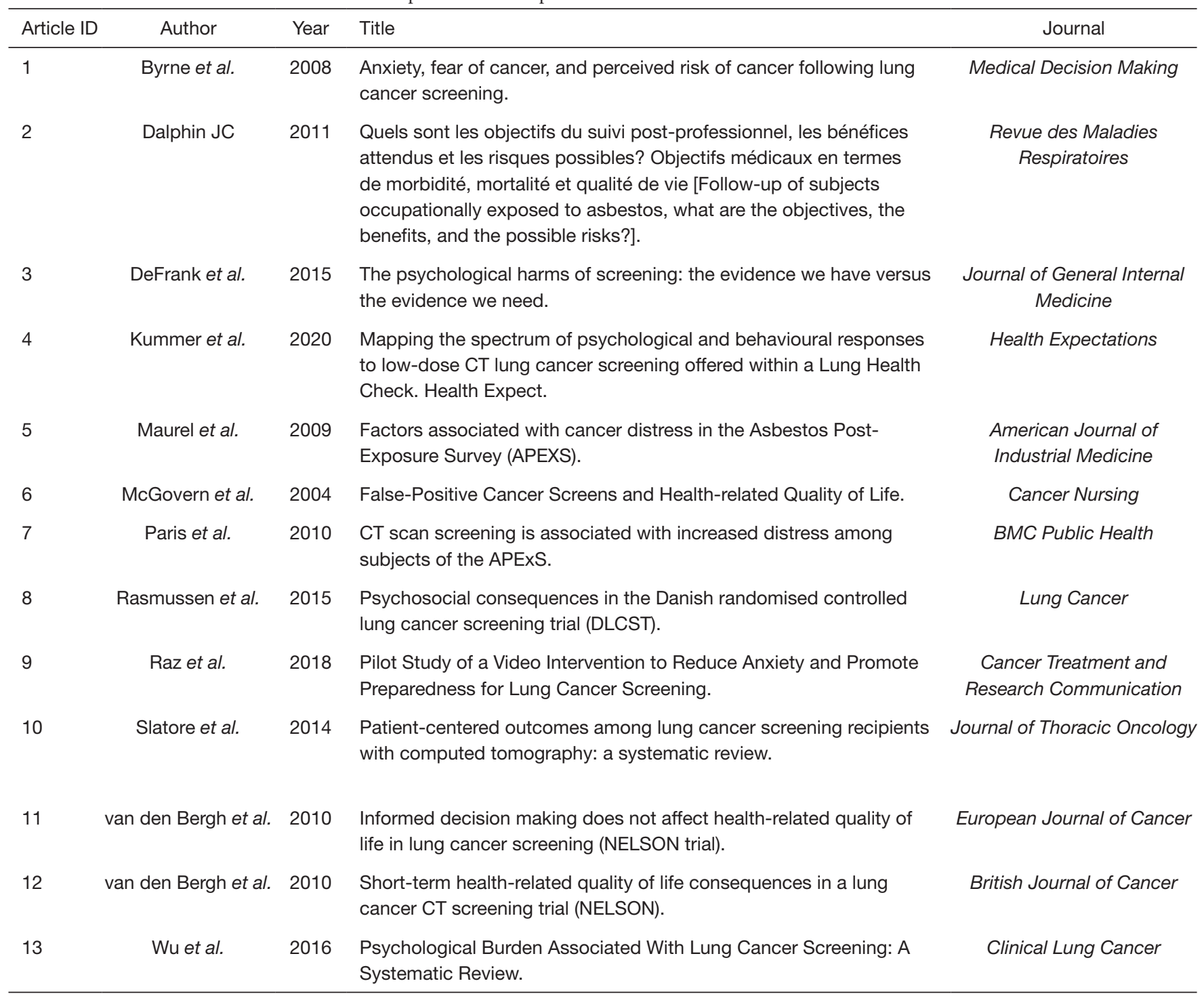

